\title{
Anomalías dento maxilares y factores asociados en niños con parálisis cerebral
}

\author{
LIVIA BARRIONUEVO N. ${ }^{1}$, FRESIA SOLÍS F. ${ }^{2}$ \\ 1. Cirujano Dentista, Unidad de Odontopediatría. Departamento Médico, Instituto de Rehabilitación Infantil \\ Teletón-Santiago. Chile. \\ 2. Tecnólogo Médico, Magíster Bioestadística, Dirección académica, Instituto de Rehabilitación Infantil Teletón-Santiago, Chile.
}

\begin{abstract}
Dento-maxillary analysis and related factors in children with Cerebral Palsy

Background: Patients with cerebral palsy attending the Children Rehabilitation Institute (Teleton) at Santiago-Chile present open bite and oral disfunctions. Objective: Determine dento-maxillary anomalies related to cerebral palsy and factors associated to feeding, breathing and parafunctional habits. Method: Descriptive study including 80 children of both sex, between 4-12 years-old, with cerebral palsy. A revision of clinical files, extra and intraoral exams and a parents investigation was performed. Results: $83 \%$ of children have severe spastic tetraparesis. A high incidence of open bite incisors was found, long faces compatible with dolicofacial growing and labial incompetence. Associated to this facial biotype, mixed breathing with bucal predominance and absence of lateral movements were found in these children. The intermaxillary relation is type I and the Angle's classification is neutron occlusion. In relation to feeding habits, $50 \%$ of patiens with tetraparesis eat their food cut in very small pieces, whereas $60 \%$ with diplegia and hemiparesia eat their food in a normal way. The parafunctional habits were significant $(47 \%)$. Conclusions: Cerebral palsy is an important condition in the development of a dento-maxillary disarmony, like open bite and dysfunctional sthomatognatic system.

(Key words: Cerebral palsy, dysfunctional sthomatognatic system, parafunctional habits, dento-maxillary anomalies)

Rev Chil Pediatr 2008; 79 (3): 272-280

\section{RESUMEN}

Introducción: Los pacientes con parálisis cerebral atendidos en el Instituto de Rehabilitación Infantil de Santiago, presentan mordidas abiertas y disfunción oral. Objetivos: Determinar anomalías dento maxilares y relacionarlas con diagnóstico de parálisis cerebral y factores asociados de alimentación, respiración y hábitos parafuncionales. Pacientes y Método: Estudio descriptivo transversal, que incluyó revisión de fichas clínicas 2005-2006, examen extra e intraoral y encuesta a los padres de 80 niños, ambos sexos, con diagnóstico de parálisis cerebral entre 4 y 12 años de edad. Resultados: 83\% de los niños, presentó
\end{abstract}

Trabajo recibido el 27 de noviembre de 2007, devuelto para corregir el 25 de enero de 2008, segunda versión el 11 de marzo de 2008, aceptado para publicación el 14 de abril de 2008.

E-mail: livia.ban@gmail.com; Livia Barrionuevo@teletón.cl 
tetraparesia espástica severa. Se encontró alta incidencia de mordida abierta anterior, caras alargadas compatibles con crecimiento dolicofacial e incompetencia labial. Asociado a este biotipo facial, se halló respiración mixta de predominio bucal y ausencia de movimientos de lateralidad. La relación intermaxilar es tipo I y neutro oclusión según clasificación de Angle. De los hábitos de alimentación, el 50\% de los pacientes con tetraparesia comen picado; alrededor del 60\% con diplejia y hemiparesia comen entero. Los hábitos parafuncionales no fueron significativos, $47 \%$ de los pacientes encuestados los tenían. Conclusiones: La parálisis cerebral es una variable importante asociada al desarrollo de una desarmonía dentomaxilar como la mordida abierta y de la disfunción del sistema estomatognático.

(Palabras clave: parálisis cerebral infantil, disfunción oral, anomalía dento maxilar, hábitos parafuncionales). Rev Chil Pediatr 2008; 79 (3): 272-280

\section{Introducción}

La parálisis cerebral (PC) se caracteriza por una lesión no progresiva en un cerebro inmaduro, en período prenatal o post natal, antes de los 5 años de edad es decir, antes que el sistema nervioso central complete su desarro1lo. No corresponde a este diagnóstico las encefalopatías producidas en edades más tardías. La característica de la PC es el trastorno del movimiento y de la postura. Junto con la alteración motora se pueden encontrar una serie de discapacidades que afectan al individuo que la padece: retardo mental, alteraciones sensoriales, de lenguaje, de aprendizaje, síndromes convulsivos, frecuentes episodios de enfermedades del tracto nasofaríngeo y respiratorio, problemas emocionales y anomalías dento maxilares $(\mathrm{ADM})^{1}$.

La gran mayoría de pacientes con PC que se atienden en el Instituto de Rehabilitación Infantil Teletón de Santiago, presentan como ADM, mordida abierta anterior, crecimiento facial vertical compatible con crecimiento dolicofacial e incompetencia labial. Además, presentan trastornos en las funciones normales del sistema estomatognático:

a) Alimentación: No son capaces de comer entero y se les cae parte de la comida de la boca.

b) Respiración: Respiran por la boca lo que les genera irritación de la mucosa bucal y de las vías respiratorias, poco desarrollo de la nariz y huesos maxilares superiores: tienen aspecto de pómulos achatados, falta humidificación y temperatura del aire inspirado, condicionando cuadros de infección respiratorios. c) Lenguaje: Tienen dificultad en la articulación de fonemas.

d) Salivación: No son capaces o tienen dificultad para tragar su saliva, lo que obliga a muchos de ellos a usar baberos, manteniendo la piel perioral irritada.

Este sistema tiene una especialización precoz: el niño nace con la región oral preparada para las funciones de succión y respiración; con la erupción de los primeros dientes temporales el niño comienza a prepararse para una nueva forma de alimentación: la masticación la cual se desarrolla definitivamente alrededor de los 18 meses con la erupción del primer molar temporal ${ }^{2}$.

La región oral tiene un componente genético en su crecimiento y desarrollo, pero la función muscular derivada de las acciones que allí se ejercen es responsable de la calidad de la musculatura insertada en esa zona y de la arquitectura (anatomía) final del sistema estomatognático ${ }^{3-5}$.

Los factores medioambientales que dañan el sistema estomatognático en niños con PC son:

1) La alteración del tono muscular que estos pacientes presentan y la postura corporal alterada que ellos adoptan.

2) Hábitos parafuncionales como succión de dedo y/o chupete, que exacerban las anomalías que puedan presentar.

3) Hábitos de alimentación, en relación a la consistencia de los alimentos versus edad cronológica $^{6}$.

Como en Chile no se encuentran estudios en niños con PC sobre hábitos para-funcionales ni 
tampoco de anomalías dento-maxilares y la rehabilitación que estos pacientes reciben actualmente sólo se refiere a prevención y tratamiento de caries y enfermedad de encías, lo que limita las comparaciones con resultados previos, se planteó como objetivo principal, realizar un diagnóstico del sistema estomatognático en niños con parálisis cerebral entre 4 y 12 años, de ambos sexos, relacionándolo con la expresión de parálisis cerebral y cuantificar la relación entre ambos maxilares y con el macizo cráneo facial. Como objetivo secundario, relacionar las diferentes expresiones de PC con hábitos para-funcionales (succión chupete y/o dedo), tipo de respiración, alimentación y movimiento de lateralidad mandibular.

\section{Pacientes y Método}

Se realizó un estudio descriptivo transversal, con base en una búsqueda en el sistema informático del Instituto para individualizar el universo de casos con parálisis cerebral. De esta población, cumplieron los requisitos de inclusión 80 niños con diagnóstico de parálisis cerebral, entre 4 y 12 años de edad, de ambos sexos, con residencia en la Región Metropolitana. Los pacientes acudieron a control a la clínica odontológica del IRI Teletón de Santiago, entre noviembre 2005 y noviembre 2006 excluyéndose: niños de otras edades, con síndromes genéticos, enfermedades metabólicas, distrofias musculares y otras enfermedades que no eran parálisis cerebral.

Las variables clínicas en relación al diagnóstico de PC se obtuvieron directamente de la ficha clínica del IRI. Las características extraorales, aspecto facial y relación intermaxilar, se registraron por observación directa del paciente. Las características intra-orales se clasificaron utilizando espejo dental y por observación directa: tipo de dentición: mixta, temporal, permanente; relación intermaxilar transversal: normal, vis a vis o invertida; relación intermaxilar sagital canina y molar: mesio, neutro o disto según clasificación de Angle; relación intermaxilar vertical: escalón normal, mordida cubierta, mordida invertida.

La variable funcional de lateralidad se logró solicitando al menor realizar el movimiento y/o moviendo manualmente el maxilar inferior. El cierre labial se observó en forma directa y solicitando al niño cierre labial. Tipo de respiración se obtuvo por observación directa y por encuesta a los padres. Las variables de alimentación, consistencia de alimentos, botar parte de la comida y de hábitos de succión de dedo y/o chupete, se obtuvieron por encuesta a los padres. Para determinar la consistencia de los alimentos, se usaron términos como licuado, picado y entero. La consistencia de picado, se refirió al trozo pequeño cortado con cuchillo y el entero a la capacidad de comer un trozo de carne, fruta o verdura cruda del tamaño de un dado.

Se elaboró una base de datos en planilla Excel y se procesó mediante software SPSS versión 14.0. El análisis estadístico incluyó: descripción de variables y pruebas basadas en distribución $\chi^{2}$ para determinar asociación entre variables con $\mathrm{p}<0,05$.

\section{Resultados}

De los 80 casos estudiados, $45 \%$ correspondió al sexo femenino, con un rango de edad para ambos sexos entre 6 y 6,9 años.

El diagnóstico de parálisis cerebral, registró $51,3 \%$ de tetraparesia y de éstas, la categoría espástica con compromiso motor severo representó el 83,3\%. Un 33,8\% correspondió a diplejia, de las cuales $73,7 \%$ fue de tipo espástica con compromiso motor moderado. El 15\% correspondió a hemiparesia (no se consignó lado derecho o izquierdo), de las cuales $50 \%$ tuvo compromiso severo y $33,3 \%$ leve.

En relación a función cognitiva, más de la mitad de los niños no la tenía consignada en ficha clínica; esta variable se mide después de los 6 años y el promedio de edad de este estudio es de 6 a 6,9 años, razón por la cual no pudo ser determinada.

El examen extraoral identificó al 71,3\% de los niños como dolicofacial independiente de su diagnóstico neurológico-topográfico. Al relacionar el diagnóstico de PC con el examen facial frontal, no hubo significación estadística pero como se muestra en tabla 1 , del total de niños 
Tabla 1. Examen funcional oral por diagnóstico de parálisis cerebral neurológica-topográfica

\begin{tabular}{|c|c|c|c|c|c|c|c|}
\hline \multirow[t]{3}{*}{ Examen funcional oral } & \multicolumn{7}{|c|}{ Diagnóstico parálisis cerebral neurológico-topográfico } \\
\hline & \multicolumn{2}{|c|}{ Hemiparesia } & \multicolumn{2}{|c|}{ Diplejia } & \multicolumn{2}{|c|}{ Cuadriparesia } & \multirow{2}{*}{$\begin{array}{c}\text { Total } \\
\text { n }\end{array}$} \\
\hline & $\mathbf{n}$ & $\%$ & $\mathbf{n}$ & $\%$ & $\mathbf{n}$ & $\%$ & \\
\hline Total muestra & 12 & 15,0 & 27 & 33,8 & 41 & 51,2 & 80 \\
\hline \multicolumn{8}{|l|}{ Movimiento lateralidad* } \\
\hline $\mathrm{Si}$ & 5 & 17,2 & 15 & 51,7 & 9 & 31,1 & 29 \\
\hline No & 7 & 13,7 & 12 & 23,5 & 32 & 62,7 & 51 \\
\hline \multicolumn{8}{|l|}{ Tipo de respiración** } \\
\hline Nasal & 4 & 36,4 & 5 & 45,5 & 2 & 18,1 & 11 \\
\hline Mixta & 8 & 11,6 & 22 & 31,9 & 39 & 56,5 & 69 \\
\hline \multicolumn{8}{|l|}{ Cierre labial** } \\
\hline $\mathrm{Si}$ & 5 & 26,3 & 9 & 47,4 & 5 & 26,3 & 19 \\
\hline No & 7 & 11,5 & 18 & 29,5 & 36 & 59,0 & 61 \\
\hline
\end{tabular}

* Significativo $p=0,01 ; * *$ Significativo $p=0,028$

con cuadriparesia, $82,9 \%$ correspondió a dolicofaciales. En los grupos con diplejia y hemiparesia se encontró este tipo de crecimiento en un $58 \%$ aproximadamente (figura 1 ).

El $62,5 \%$ de los casos correspondió a clase I, con una relación de ambos maxilares dentro de lo normal. En el examen intraoral no se observó compresión maxilar y la mordida abierta anterior se presentó en $63,8 \%$ de los pacientes. La relación canina fue un $47 \%$ de los casos neutra según Angle, pero en un $25 \%$ no fue posible determinar su relación porque los caninos estaban en inoclusión. Dado el rango de edad de los pacientes se observó 70\% dentición de tipo mixta.

En cuanto al examen funcional oral, se observó que el $63,8 \%$ no era capaz de realizar lateralidad; $86,2 \%$ tenía respiración mixta de predominio bucal y $73,2 \%$ presentó incompetencia labial (figura 2).

$\mathrm{Al}$ relacionar los tópicos anteriores: movimiento de lateralidad, tipo de respiración y cierre labial con el diagnóstico de PC, se registró alta significación estadística $(\mathrm{p}<0,05)$. En el grupo de las tetraparesias, no presentaron lateralidad el $62,7 \%$; respiración mixta de predominio bucal en 56,5\% y sin cierre labial un 59,0\%.

Es importante notar que en los pacientes con diplejia y hemiparesia, estas variables estuvieron dentro de límites normales. Sin embargo, en los niños diagnosticados con diplejia, se registró ausencia de movimiento de lateralidad en $23,5 \%$; respiración mixta en $31,9 \%$ y sin cierre

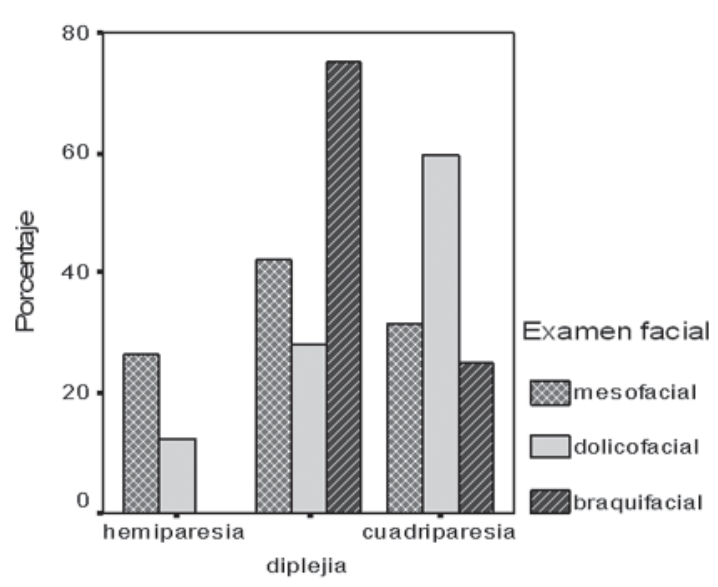

Figura 1. Examen facial frontal por diagnóstico de PC neurológico-topográfico.

labial en 29,5\%; En los niños con hemiparesia se obtuvo que $13,7 \%$ no pudo realizar lateralidad, $11,6 \%$ tuvo respiración mixta y $11,5 \%$ no cerraban los labios (tabla 1).

En cuanto a los hábitos parafuncionales como succión de chupete y/o dedo, se presentó en $55 \%$ de los casos, con una duración hasta los 7 años en $33,8 \%$ de los casos.

La relación examen funcional y hábitos parafuncionales, registró significación estadística sólo con tipo de respiración, predominando respiración mixta en $51,2 \%$ de todos los casos. El resto de las funciones orales estudiadas no 

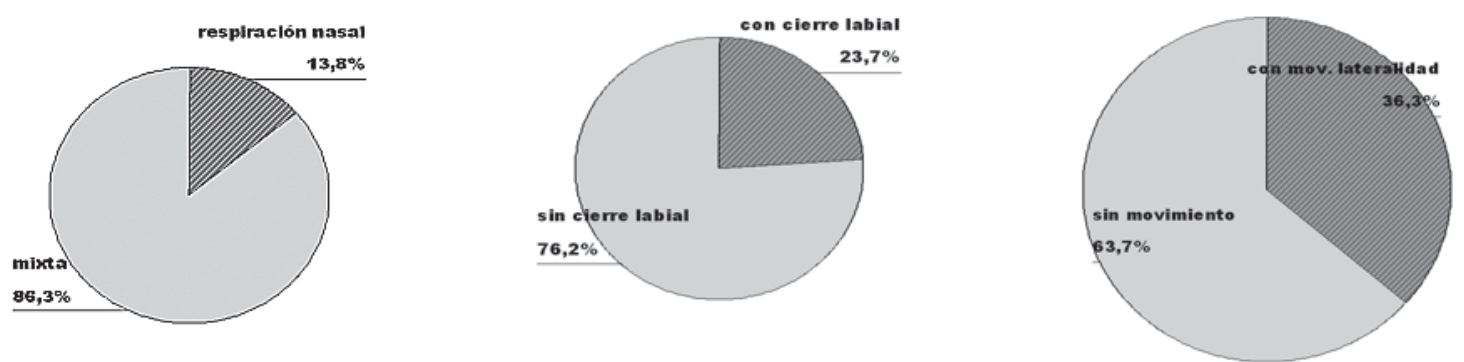

Figura 2. Examen funcional oral: tipo de respiración, competencia labial y movimiento de lateralidad.

Tabla 2. Hábito oral parafuncional por examen funcional oral, dirección de crecimiento facial y relación oclusal vertical

\begin{tabular}{|c|c|c|c|c|c|}
\hline \multirow[t]{2}{*}{ Examen funcional oral } & \multicolumn{5}{|c|}{ Hábito oral para-funcional } \\
\hline & si & $\%$ & no & $\%$ & Total \\
\hline Total muestra & 44 & 55,0 & 36 & 45,0 & 80 \\
\hline \multicolumn{6}{|l|}{ Movimiento laterall } \\
\hline $\mathrm{Si}$ & 15 & 51,7 & 14 & 48,3 & 29 \\
\hline No & 29 & 56,9 & 22 & 43,1 & 51 \\
\hline \multicolumn{6}{|l|}{ Tipo de respiración ${ }^{2}$} \\
\hline Nasal & 3 & 27,3 & 8 & 72,7 & 11 \\
\hline Mixta & 41 & 59,4 & 28 & 40,6 & 69 \\
\hline \multicolumn{6}{|l|}{ Cierre labia $\beta$} \\
\hline $\mathrm{Si}$ & 8 & 42,1 & 11 & 57,9 & 19 \\
\hline No & 36 & 59,0 & 25 & 41,0 & 61 \\
\hline \multicolumn{6}{|l|}{ Examen facial fronta ${ }^{\beta}$} \\
\hline Braquifacial & 10 & 52,6 & 9 & 47,4 & 19 \\
\hline Dolicofacial & 31 & 54,4 & 26 & 45,6 & 57 \\
\hline Mesofacial & 3 & 75,0 & 1 & 25,0 & 4 \\
\hline \multicolumn{6}{|l|}{ Relación oclusal verticap } \\
\hline Escalón normal & 12 & 42,9 & 16 & 57,1 & 28 \\
\hline Aumentada & 1 & 100,0 & - & - & 1 \\
\hline Invertida o mordida abierta anterior & 31 & 60,8 & 20 & 39,2 & 51 \\
\hline
\end{tabular}

${ }^{1}$ no significativo $p=0,657 ;{ }^{2}$ significativo $p=0,045 ;{ }^{3}$ no significativo $p=0,196 ;{ }^{4}$ no significativo $p=0,715 ;{ }^{5}$ no significativo $\mathrm{p}=0,141$

fueron significativas con succión de chupete y/o dedo (tabla 2).

$\mathrm{Al}$ asociar la consistencia de los alimentos con el diagnóstico de PC se observó relación significativa entre ambas variables $(p<0,05)$. De los niños que comían picado, $77,3 \%$ tenía cuadriparesia y de los que comían entero, $64 \%$ presentaba diplejia y $24 \%$ hemiparesia, comportamiento esperable dado el compromiso motor.
La variable saca la lengua/bota parte de la comida estuvo presente en todos los diagnósticos de PC pero con alto porcentaje en las tetraparesias (57,5\%). Cabe consignar que el $15 \%$ de los niños estaban gastrostomizados, 2 de ellos con diagnóstico de hemiparesia y 10 diagnósticos de cuadriparesia. Los hábitos parafuncionales, estaban presentes en un porcentaje preocupante en todos los diagnósticos de PC, aunque la relación no fue significativa (tabla 3). 
Tabla 3. Consistencia de alimentos y hábitos de alimentación según diagnóstico de parálisis cerebral neurológico-topográfico

\begin{tabular}{|c|c|c|c|c|c|c|c|}
\hline \multirow{3}{*}{$\begin{array}{l}\text { Consistencia } \\
\text { alimentos y hábitos }\end{array}$} & \multicolumn{7}{|c|}{ Diagnóstico parálisis cerebral neurológico-topográfico } \\
\hline & \multicolumn{2}{|c|}{ Hemiparesia } & \multicolumn{2}{|c|}{ Diplejia } & \multicolumn{2}{|c|}{ Cuadriparesia } & \multirow{2}{*}{$\begin{array}{c}\text { Total } \\
\mathbf{n}\end{array}$} \\
\hline & $\mathbf{n}$ & $\%$ & $\mathbf{n}$ & $\%$ & $\mathbf{n}$ & $\%$ & \\
\hline \multicolumn{8}{|l|}{ Consistencia alimentos? } \\
\hline Licuado + colado & 2 & 9,5 & 8 & 38,1 & 11 & 52,4 & 21 \\
\hline Picado & 2 & 9,1 & 3 & 13,6 & 17 & 77,3 & 22 \\
\hline Entero & 6 & 24,0 & 16 & 64,0 & 3 & 12,0 & 25 \\
\hline Total & 10 & 14,7 & 27 & 39,7 & 31 & 45,6 & 68 \\
\hline \multicolumn{8}{|l|}{$\begin{array}{l}\text { Saca lengua/bota parte } \\
\text { de la comida }\end{array}$} \\
\hline $\mathrm{Si}$ & 2 & 6,1 & 12 & 36,4 & 19 & 57,5 & 33 \\
\hline No & 8 & 22,9 & 15 & 42,9 & 12 & 34,3 & 35 \\
\hline Gastrostomizado & 2 & 16,7 & - & - & 10 & 83,3 & 12 \\
\hline Total & 12 & 15,0 & 27 & 33,8 & 41 & 51,2 & 80 \\
\hline \multicolumn{8}{|c|}{ Hábitos orales parafuncionales ${ }^{3}$} \\
\hline $\mathrm{Si}$ & 8 & 18,2 & 15 & 34,1 & 21 & 47,7 & 44 \\
\hline No & 4 & 11,1 & 12 & 33,3 & 20 & 55,6 & 36 \\
\hline Total & 12 & 15,0 & 27 & 33,8 & 41 & 51,2 & 80 \\
\hline
\end{tabular}

${ }^{1}$ significativo $p<0,01 ;{ }^{2}$ significativo $p=0,012 ;{ }^{3}$ no significativo $p=0,638$

\section{Discusión}

Si bien es cierto varios autores han encontrado una gran incidencia de anomalías dento maxilares y disfunción oral en niños con parálisis cerebral tales como: mordidas abiertas esqueletales, crecimiento dolicofacial, mordidas clase II esqueletal, disfunción articulación témporo mandibular, asimetrías faciales e incompetencia labial ${ }^{7,8}$, este estudio reafirma que, la parálisis cerebral, en sus formas tetraparesia, diplejia o hemiparesia, es una variable importante asociada al desarrollo de una anomalía dento maxilar, especialmente la mordida abierta, es decir, con el progreso de la edad se va produciendo un deterioro de las funciones de este sistema, identificado también por Pelegano en 1994 ${ }^{\circ}$ La relación intermaxilar fue clase I según clasificación de Angle y no se observó compresión maxilar superior, ni clase II, como en otros estudios $^{10,11}$. La ausencia de concordancia con otros autores, podría deberse al rango de edad considerado, de 4 a 12 años. Hemos observado que en adolescentes de 14 años ya existe una mordida abierta esqueletal con tope molar, relación intermaxilar clase II y compresión maxilar, en los cuales la lengua es un factor fundamental en el desarrollo de la ADM, pues al tener su dinámica alterada, no se apoya en el paladar en posición de reposo, ubicándose permanentemente en el maxilar inferior. Por otra parte, la lengua tiene tamaño normal pero su continente, los maxilares, están pocos desarrollados, lo que favorece que la lengua se interponga entre ellos, incrementando la mordida abierta. Sería conveniente realizar estudios para realizar un diagnóstico precoz de las maloclusiones $^{12}$ y determinar la edad que comienza a transformarse una mordida abierta anterior en mordida abierta con extensión a molares, para implantar medidas preventivas en este tipo de pacientes. Los aspectos funcionales de respiración $(86,2 \%$ mixta), cierre labial (ausente en $73,2 \%$ ) y movimiento de lateralidad mandibular (ausente en 63,8\%) están profundamente alterados en niños con PC, principalmente en aquellos con tetraparesia; en un porcentaje menor en pacientes con diplejia y cerca del $12 \%$ en niños con hemiparesia, lo que indicó relación directa y significativa con el diagnóstico neurológico de PC y su severidad. Al no tener lateralidad mandibular, estos niños tienen problemas masticatorios.

La masticación, en condiciones normales, se adquiere mediante el logro gradual de los movimientos que la compone: apertura y cierre 
y lateralidad o molienda, este último aparece alrededor de los 18 meses y madura alrededor de los dos años y medio con la presencia de una dentición temporal completa. Este grupo de niños, realizan la masticación mayoritariamente con movimiento de bisagra, no existiendo una molienda real, razón por la cual se alimentan con consistencia picado o molido con tenedor. Coincidimos con otras investigaciones en relación a que los factores determinantes no están muy claros, pero pueden ser una asociación de alteración en el tono muscular, falta de actividad oral, retardo mental, falta de madurez psicomotora, disfunción respiratoria, lingual u oclusal o falta de estímulo por parte de los padres ${ }^{13-15}$.

Si bien los hábitos de succión no mostraron ser significativos en el desarrollo de ADM, es importante notar que están presentes en 56,9\% de los niños que no tienen lateralidad, en $59 \%$ de los niños con incompetencia labial, en $54,4 \%$ de los niños dolicofaciales y en $30 \%$ de los pacientes con mordida abierta. Los hábitos parafuncionales, fueron significativo en relación a la respiración de tipo mixta, lo mismo que en niños $\sin \mathrm{PC}^{16}$. Al existir respiración de tipo mixta, el cierre labial se altera, situación registrada en pacientes con PC aunque sin significación estadística. Extrapolando lo que implica una respiración de tipo mixta, en donde está presente la mordida abierta, escaso desarrollo del tercio medio de la cara, un maxilar alto y comprimido, alteración de la dinámica lingual y posición baja de la misma, constante irritación del sistema respiratorio, se puede deducir que se intensifica el crecimiento vertical o dolicofacial $^{17}$, hecho observado en este estudio pero sin significación estadística.

La succión de chupete es el hábito parafuncional más prevalente en estos pacientes, más allá de los dos años. El chupete es un elemento pacificador pero, hoy se usa como juguete, permanece constantemente prendido de la ropa del niño, al alcance de él, lo que favorece el uso del mismo por parte del menor, quien dado la edad o la inmadurez, no es capaz de discriminar cuando usarlo o no. Se puede apreciar tanto en niños con PC como en niños sin PC. El problema más grave en relación a la succión de chupete/dedo, es la alteración de la dinámica lingual. En una lengua cuyo tono está alterado por la PC, la interposición de un objeto, hace que ésta se ubique permanentemente en el maxilar inferior y nunca sube a apoyarse en el maxilar superior. La lengua es un potente músculo que estimula el crecimiento y desarrollo del maxilar superior. Si no está en contacto con el paladar, trae como consecuencia una bóveda palatina poco desarrollada (compresión maxilar) y un incremento en la mordida abierta. La reeducación de la lengua necesita una activa participación y entendimiento del paciente lo que no se encuentra siempre en un niño con PC. De esto se deduce la importancia que se debe dar a la información, por nuestra parte, a los padres y del conocimiento que el equipo médico debe tener en relación a la alteración de la dinámica lingual versus succión de chupete.

La consistencia de la alimentación, que se traduce en eficiencia masticatoria, no fue significativa en este grupo de niños. Sin embargo, llamó la atención que niños con hemiparesia coman entero sólo en un $24 \%$ y niños con diplejia lo hagan en un $64 \%$, es posible que exista un retardo mental o un trastorno conductual de alimentación. La excesiva protección materna de los menores, hace que estos niños mantengan conductas orales infantiles por largo períodos lo que impide que ellos realicen las funciones normales de masticación. Al favorece hábitos infantiles, como alimentación licuada hasta pasado los tres o cuatro años, se genera, problemas de tipo conductual, es decir, sin tener ningún síntoma o signo de un trastorno de deglución se comportan como si éste existiera. Esta conducta podría ser una de las causas por la cual niños con daño neurológico moderado o leve tienen las mismas características oro faciales que un niño con daño severo. Es importante aclarar este punto, ya que la masticación es una de las funciones que desarrolla la musculatura masticatoria, y de ésta capacidad va a depender la modelación ósea del sistema estomatognático y de las funciones que allí se ejercen. La importancia de la consistencia del alimento radica en que los receptores ubicados en el periodonto del diente envían la información, vía aferente al SNC, cuya eferencia determina la fuerza de contracción muscular y el movimiento masticatorio a realizar ${ }^{18}$. 
La caída de saliva o babeo, no fue examinada en este trabajo, sin embargo, fue observa$\mathrm{da}$ con frecuencia en estos pacientes y, pareciera ser consecuencia de una dificultad en tragar la saliva, en cerrar los labios, en el cierre bucal por mordida abierta severa o todas en el momento de deglutir, y no en un aumento de la producción de ésta por parte de las glándulas salivales $^{19,20}$. Se requiere estudio adicional al respecto.

Existe un número pequeño, pero no menos importante de pacientes con PC tipo tetraparesia, diplejia o hemiparesia con compromiso leve y con un coeficiente intelectual normal o bajo, que también desarrollan este tipo de ADM y que genera en ellos un problema de relación con sus pares por el aspecto facial que presentan: cara larga de poco inteligentes, boca permanentemente abierta y todas las disfunciones orales ampliamente mencionadas: babeo, protrusión lingual al comer con caída del alimento, dificultad para pronunciar algunos fonemas, etc en este grupo es donde deben implantarse medidas preventivas y cuando el daño se ha presentado deben ser rehabilitados en forma integral para que logren una inserción social, educacional y laboral real.

Aunque lo anteriormente expuesto es conocido, con abundante literatura extranjera al respecto, en nuestro país no lo habíamos cuantificado hasta hoy. Conocer cómo se desarrolla una anomalía dento maxilar, tener un diagnóstico orofacial y determinar las alteraciones morfofuncionales que los pacientes con parálisis cerebral presentan, nos permite implementar o crear en un futuro próximo, medidas preventivas, curativas o ambas de estas alteraciones en la edad que corresponda. La rehabilitación del sistema estomatógnatico de maduración precoz, debe comenzar en edades tempranas, partiendo desde la lactancia materna $^{21}$, por lo tanto, es importante educar a padres y personal que colabora en la rehabilitación oral de estos pacientes, en relación a su crecimiento y desarrollo, hábitos de alimentación y para-funcionales. Con este trabajo pretendemos sentar un antecedente para estimular a otros a continuar con la investigación y/o a crear sistemas de prevención y tratamientos.

\section{Conclusiones}

La parálisis cerebral es una variable asociada a la génesis de anomalía dentomaxilar y el daño depende de la gravedad de la lesión cerebral.

Se verifica mayor frecuencia de alteración de la función en el sistema estomatógnático y crecimiento vertical o dolicofacial en pacientes con tetraparesia.

Las características anátomo-funcionales del sistema estomatognático están alteradas en directa relación al daño neurológico que el niño presente.

Los hábitos de succión dedo/chupete, alimentación molida con tenedor o licuada y respiración bucal exacerban las alteraciones morfo funcionales de este sistema.

Se requiere realizar más estudios, de tipo longitudinal, comenzando en edades muy tempranas, apenas diagnosticada la PC.

\section{Referencias}

1.- Mena M: Síndrome de parálisis cerebral. En: Enfermedades invalidantes de la infancia. Enfoque integral de rehabilitación vol II pág14-19. Santiago. IVROS 2006.

2.- Schellhorm C: Control odontológico del niño sano. Santiago. Atos.1992. 112p.

3.- Enlow D Mark H: Crecimiento facial, México, Mac Graw-Hill. 1998. 320 p.

4.- Vellini F: Ortodoncia. Diagnóstico y planificación clínica. $3^{\text {a }}$ edición, Sao Paulo, Artes Médicas. 2002.

5.- Ruiz I, Alvarez A, González J: Embriología facial: diagnóstico de las principales malformaciones congénitas. En: Manual de cirugía plástica. Www.secpre.org/ documentos\%20manual\%2021.html

6.- Navas CX: Trastornos del mecanismo succión deglución. Rev Gastrohnup 2003; 5 (1): 73-5.

7.- Franklin DL, Luther F, Curzon MEJ: The prevalence of malocclusion in children with cerebral palsy. European Journal of Orthodontic 1996; (18): 63743.

8.- Alfaro P, Osorno MC, Sánchez L, Sáenz LP: Relación entre oclusión dental y función de la articulación temporomandibular en niños con parálisis cerebral. www.xoc.uam.mx/temas_selectos/relación_entre oclusión_de.htm-28k. (Enero 2006).

9.- Pelegano J, Nowysz S, Goepferd S: Temporomandibular joint contracture in spastic quadriplegia: effect on oral motors skills. Developmental Medicine and Child Neurology 1994; 36: 487-94.

10.- Strodel BJ: The effects of spastic cerebral palsy on occlusion. J Dent Child 1987; 54 (4): 255-60. 
11.- Oreland A, Heijbel I, Jagell S: Malocclusion in phisiclally and/or mentally handicapped children. Swed Dent J 1987; 11 (3): 103-19.

12.- Vela A: Diagnóstico precoz de las maloclusiones esqueléticas y dentales en la infancia. www.dentopolis. com $/$ modules.php? name $=$ Content\&Ortodoncia $($ Enero 2006).

13.- Argandoña J: Sistema estomatognático en niños. Desarrollo, función y disfunción. Revista Sociedad Chilena de Odontopediatría 2003. Ed. especial. vol 15 y16: 19-22.

14.- Vittek J, Winik S, Winik A, et al: Analysis of orthodontic anomalies in mentally retarded developmentally disable (MRDD) persons. Spec care dentist 1994; 14 (5): 198202.

15.- Vera A, Chacón E, Ulloa R: Estudio de la relación entre la deglución atípica, mordida abierta, dicción y rendimiento escolar por sexo y hogar en niños de preescolar a sexto grado en dos colegios de Catia. http://www.ortodoncia.ws/publicaciones/2004deglucion atipica_mordida_abierta_diccion_rendimiento_escolar.asp $-114 \mathrm{k}$

16.- Agurto P, Díaz R, Cádiz O, Bobenrieth F: Frecuencia de malos hábitos orales y su asociación con el desarrollo de anomalías dentomaxilares en niños de 3 a 6 años de área Oriente de Santiago. http://www.scielo.cl/ scielo.php?pid=S0370-1061999000600004\&script $=$ sci_arttext\&tlng=es (Nov. 2005).

17.- Garrido G: El crecimiento vertical. Etiología, diagnóstico y tratamiento en dentición mixta. Publicación Fundaciones Gnathos. http://w.w.w.gnathos.net/ administrador/artículos_científicos/upload/28.pdf

18.- La ortodoncia del siglo XXI. Revista de Odontología. http://encolombia.com/odontologia/investigaciones/ marilhtm-10k

19.- Schwartz S, Gisl EG, Clarke D, Haberfellner H: Association of occlusion with eating efficiency in children with cerebral palsy and moderata eating impairment. J Dent Child 2003; 70 (1): 33-9.

20.- Tahmassebi JF, Luther F: Relationship between lip position and drooling in children with cerebral palsy. Paediatr Dent 2004; 5 (3): 151-6.

21.- Pilonieta G, Torres E: Implicaciones de la lactancia materna en odontopediatría. MEDUNAB. 2003. 6(17): 89-91. http://editorial.unab.edu.co/revistas/medunab/ rev617.html. (Marzo 2006). 